\title{
Marked extension of proliferation of rat Sertoli cells in culture using recombinant human $\mathrm{FSH}$
}

\author{
J. J. Buzzard ${ }^{1,2}$, N. G. Wreford ${ }^{1}$ and J. R. Morrison ${ }^{2 *}$ \\ ${ }^{1}$ Department of Anatomy and Cell Biology and ${ }^{2}$ Monash Institute of Reproduction and \\ Development, Monash University, Clayton 3168, Melbourne, Australia
}

Previous studies indicate that proliferation of rat Sertoli cells in culture can only be maintained until the equivalent of days 10-12 after birth, irrespective of the age of the donor animal. This report describes methods for the isolation and culture of Sertoli cells from day 6 rat testes, which can proliferate in culture for 20-24 days (that is, until the equivalent of days 26-30 after birth). Cells were isolated by enzymatic digestion of seminiferous cords followed by selective depletion of contaminating peritubular cells by adhesion to a polystyrene surface. The purity of the Sertoli cells was assessed using a combination of markers to be $>99.5 \%$. Proliferation was assayed using tritiated thymidine incorporation and further verified by bromodeoxyuridine histochemistry and flow cytometry. Sertoli cells proliferated at basal levels in Dulbecco's modified Eagle's medium (DMEM)-F12 media alone, and proliferation was stimulated further by addition of recombinant human FSH to the culture media. After 20-24 days in culture, proliferation rapidly ceased, and cells assumed abnormal morphology and detached from the culture vessel; these events are consistent with the cells undergoing classic rodent cell senescence. The method described provides a useful tool for investigating the control of Sertoli cell division. Furthermore, these findings indicate that the timely differentiation of Sertoli cells is not dependent solely on an intrinsic timing mechanism, as has been suggested previously.

\section{Introduction}

Sertoli cells are essential regulators of spermatogenesis, and each Sertoli cell is capable of supporting a limited number of germ cells to maturity (Orth et al., 1988). Therefore, the number of Sertoli cells present in the mature testis determines an upper limit for sperm production. In rats, Sertoli cells proliferate in the perinatal period before undergoing a process of differentiation, which includes cessation of mitosis, morphological changes and expression of several paracrine factors that support spermatogenesis. Stereological data demonstrate that, in normal rats, the number of Sertoli cells does not increase significantly after day 15 after birth (Wang et al., 1989); however, small amounts of cell division, detected by labelled nucleotide incorporation, have been reported as late as day 21 after birth (Orth, 1982; van Haaster et al., 1993). Several endocrine factors regulate the Sertoli cell proliferative phase, including FSH, a potent Sertoli cell mitogen (Orth, 1984; Meachem et al., 1996), and tri-iodothyronine, which modifies the duration of the proliferative phase. Hyperthyroidism leads to restriction of Sertoli cell proliferation until about day 12 after birth (van Haaster et al., 1993), whereas hypothyroidism leads to maintenance of Sertoli cell division until at least day 30 after birth (Cooke and

*Correspondence

Email: john.morrison@med.monash.edu.au
Meisami, 1991; van Haaster et al., 1992), albeit at a low rate.

Steinberger and Steinberger (1971) indicated that, in serum-free testis fragment cultures, Sertoli cells cease proliferation at the equivalent of days 10-12 after birth, irrespective of the age of the rat from which the cells were isolated. This finding prompted the suggestion that Sertoli cells contain an intrinsic timing mechanism that initiates their differentiation in the absence of external signalling factors. However, in addition to Sertoli cells, testis fragments contain peritubular cells, germ cells, Leydig cells, macrophages and blood vessels. It is unclear how secretions from these other testicular types of cell influence postnatal Sertoli cell development. Furthermore, Sertoli cells are normally exposed to thyroid hormone in vivo; however, in the absence of thyroid hormone, Sertoli cells are capable of proliferating for at least 30 days after birth (Cooke and Meisami, 1991; van Haaster et al., 1992). In the absence of thyroid hormone, Sertoli cells should be capable of proliferation for at least the same duration in vitro as in vivo. Clearly, it is necessary to investigate whether the cessation of Sertoli cell proliferation at days 10-12 after birth occurs in the absence of thyroid hormone and secretions from Leydig, peritubular and other testicular cells.

Studies on isolated Sertoli cells in culture have not directly addressed the duration of Sertoli cell proliferation, but have indicated that, in the presence of FSH, Sertoli cells cease proliferation after only a few days in culture (Boitani et al., 1995; Schlatt et al., 1996) in a manner similar to that 
reported by Steinberger and Steinberger (1971) in fragment culture. Furthermore, the propensity for contaminating peritubular cells, which are difficult to separate from Sertoli cells, to proliferate faster than Sertoli cells and effectively 'take-over' the culture (Schlatt et al., 1996) has made it difficult to study proliferative phase Sertoli cells in culture for more than about 7 days.

The methods presented in this report allow the study of Sertoli cell proliferation in the absence of extraneous factors produced by contaminating cells. Furthermore, ovine pituitary $\mathrm{FSH}(\mathrm{oFSH}$, a reagent routinely used as a mitogen in Sertoli cell culture experiments) is shown to be capable of inhibiting Sertoli cell proliferation. In the absence of oFSH, highly purified preparations of Sertoli cells are capable of proliferating in culture for 20-24 days (that is, to the equivalent of days 26-30 after birth). These data indicate that Sertoli cell differentiation is not controlled by an autonomous, intrinsic maturation programme, but rather that the differentiation of these cells must be influenced by factors from outside the cell.

\section{Materials and Methods}

\section{Media and culture vessels}

Dulbecco's modified Eagle's medium (DMEM)-F12 (number 12400-024) was supplemented with penicillinstreptomycin and chemically defined lipid concentrate (all from GibcoBRL Invitrogen, Melbourne) for all applications except for washing and digestion steps. This DMEM-F12 media was used alone, or further supplemented with 390 iu recombinant human $\mathrm{FSH} \mathrm{I}^{-1}$ (rhFSH media; Organon, Oss), 390 iu ovine pituitary FSH ${ }^{-1}$ (oFSH media; NIDDK-oFSH20; NIH, Bethseda, MD), insulin-transferrin-selenium concentrate (ITS; number 41400-045; GibcoBRL), or a combination of these.

Culture vessels coated with $1 \mu \mathrm{g}$ laminin $\mathrm{cm}^{-2}$ were prepared by incubation with a solution of laminin (number L2020; Sigma, St Louis, MO) in PBS (number 21600-010; GibcoBRL) for $2 \mathrm{~h}$ at $37^{\circ} \mathrm{C}$. This supernatant was aspirated and the Sertoli cells were seeded immediately. Allowing the laminin solution to dry out resulted in a marked decrease in Sertoli cell plating efficiency.

\section{Isolation of Sertoli cells}

Male Sprague-Dawley rats were obtained from the Monash Central Animal House, and were kept in accordance with the Australian Code of Practice for the Care and Use of Animals for Scientific Purposes (1997, National Health and Medical Research Council, Australia).

All Sertoli cell preparations were isolated from rats killed by decapitation 6 days after birth. Six to fourteen rats were used for each Sertoli cell preparation. Modifications to existing techniques (Steinberger et al., 1975; Risbridger et al., 1989) were required to achieve highly purified Sertoli cells from day 6 rat testes. Testes were decapsulated, diced with a razor blade until gel-like in appearance and passed through a 22-gauge needle three times. The resultant fragments of seminiferous cords (which tended to be clumped together) were rinsed in DMEM-F12 twice, and then digested with $3 \times 10^{5} \cup$ collagenase type II I-1 $^{-1}$ (number C6885; Sigma) in DMEM-F12 at $22^{\circ} \mathrm{C}$ for $1 \mathrm{~h}$. The isolated cord fragments were pelleted by centrifugation at $900 \mathrm{~g}$ for $2 \mathrm{~min}$ and rinsed twice. The cord fragments were digested with $0.05 \%(\mathrm{w} / \mathrm{v})$ hyaluronidase (number $\mathrm{H} 2126$; Sigma) in DMEM-F12 for $1 \mathrm{~h}$ at $22^{\circ} \mathrm{C}$ on a rocking platform. The cord fragments were further disaggregated by repeated pipetting and a Pasteur pipette was used to remove any remaining tissue clumps (until only single pieces of seminiferous cord were suspended in the media). The fragments were allowed to sediment at $1 \mathbf{g}$; the supernatant was discarded and the sedimented cord fragments were rinsed several times before resuspension in DMEM-F12. Cord fragments were then plated down on to culture dishes according to the degree of purity required. For high purity (98\% pure) Sertoli cells, cords were plated down on laminin-coated culture dishes with ITS with or without rhFSH. For ultra-pure (99.8\% pure) Sertoli cells, a novel method was developed whereby cord fragments were seeded on to untreated polystyrene culture flasks (BD Falcon, San Jose, CA) in media containing rhFSH but no ITS. Within 3-5 days of culture, contaminating peritubular cells adhere to the uncoated culture surface, whereas most Sertoli cells remain associated with each other in roughly spherical aggregates that are weakly adherent, or detached from the culture surface (Fig. 1a). Sertoli cell aggregates were detached from the flask by tapping; the cells were pelleted at $1 \mathbf{g}$ and seeded on to laminin-coated surfaces in the presence of ITS, where they quickly spread to form a monolayer (Fig. 1b).

In some experiments, Sertoli cells were passaged by treatment with a volume of trypsin-versene solution containing $0.1 \%(\mathrm{w} / \mathrm{v})$ trypsin (number T2021; Sigma), $0.5 \mathrm{mmol}$ tetrasodium EDTA I-1 (number E6511; Sigma) in PBS for $5 \mathrm{~min}$ at $37^{\circ} \mathrm{C}$ followed by two volumes $0.05 \%$ $(\mathrm{w} / \mathrm{v})$ soybean trypsin inhibitor (number T6522; Sigma) in DMEM-F12. Recovered cells were counted on a haemocytometer using Trypan blue exclusion to determine viability. Viable cells were rinsed and replated at $4 \times 10^{4}$ cells $\mathrm{cm}^{-2}$ (unless otherwise specified).

\section{$\left[{ }^{3} \mathrm{H}\right]$ Thymidine incorporation assay}

[Methyl- ${ }^{3} \mathrm{H}$ ] thymidine incorporation was used to measure proliferative activity. Pre-warmed [methyl- ${ }^{3} \mathrm{H}$ ] thymidine (2 $\mathrm{Ci} \mathrm{mmol}^{-1}, 3 \times 10^{5}$ c.p.m. per well; Amersham, Uppsala) in DMEM-F12 was added to the culture medium for $18 \mathrm{~h}$. Cells were rinsed and harvested with trypsin-versene solution using a Micromate 196 Cell Harvester (Packard Instruments, Meriden) by immobilizing the cells on a glass fibre filter (Packard) which was then rinsed with ethanol. Filters were air-dried, placed into $1 \mathrm{ml}$ liquid scintillant, and incorporated radionucleotide was measured using a liquid scintillation counter. 

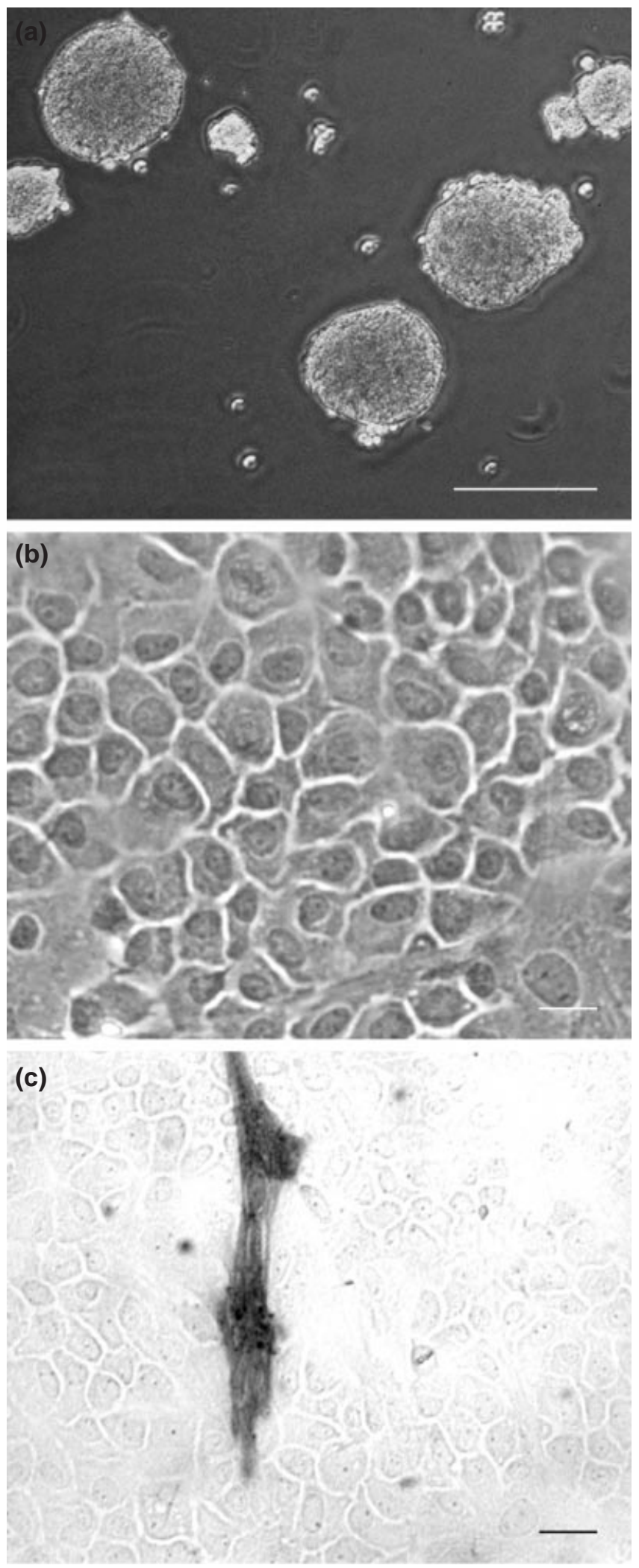

Fig. 1. Typical microscopic appearance of cultured preparations of rat Sertoli cells. (a) Phase-contrast appearance of Sertoli cell aggregates 5 days after preparation and seeding on to an uncoated polystyrene culture vessel in the presence of rhFSH. (b) Phase-contrast

\section{Immunohistochemistry}

The purity of Sertoli cells was determined by histochemistry to detect alkaline phosphatase, a cell surface marker of peritubular myoid cells, as described by Chapin et al. (1987; Fig. 1c), and verified by immunohistochemistry for $\alpha$-smooth muscle actin and inhibin $\alpha$. Purity (\%) was determined by counting all nuclei in 15 high-power light microscope fields (approximately 10-50 nuclei per field) in three separate Sertoli cell preparations and was expressed as a percentage of alkaline phosphatase-negative cells. At least 1250 cells were counted from each method to generate purity data.

In some experiments, $0.65 \mathrm{nmol} 5$-bromo-2'-deoxyuridine $\mathrm{I}^{-1}$ (BrdU, number B5002; Sigma) was added to the culture media for $2 \mathrm{~h}$ to label S-phase cells. BrdU was detected by immunohistochemistry to verify that proliferating cells were Sertoli cells and not contaminating cells. Cells were rinsed in PBS, and fixed in Bouin's fixative for $2 \mathrm{~h}$ before immunohistochemical staining. Incorporated BrdU and $\alpha-$ smooth muscle actin staining (which were done simultaneously on the same slide), were performed by treating cells with $6 \mathrm{~mol} \mathrm{HCl} \mathrm{I}^{-1}$ in PBS with $0.1 \%(\mathrm{w} / \mathrm{v})$ polyoxyethylene (10) isooctylphenyl ether (Triton X-100; Sigma) for 15 min to denature DNA, and then treating them with 0.1 mol sodium borate $\mathrm{I}^{-1}$ in PBS with $0.1 \%(\mathrm{w} / \mathrm{v})$ Triton $\mathrm{X}-100$ for $10 \mathrm{~min}$. The cells were then washed, non-specific protein binding was blocked with $10 \%$ normal goat serum in PBS, and the primary antibodies (monoclonal anti-BrdU $0.25 \% \quad(\mathrm{v} / \mathrm{v})$ and monoclonal anti- $\alpha$-smooth muscle actin $0.2 \%$ (v/v); Sigma) were incubated overnight at $4^{\circ} \mathrm{C}$. The cells were washed again and then incubated with $1.7 \%(\mathrm{v} / \mathrm{v})$ fluorescein isothiocyanate (FITC)-conjugated anti-mouse IgG (Dako, Glostrup) for $1 \mathrm{~h}$. Coverslips were mounted on glass slides using Vectashield mounting media (Vector Laboratories, Burlingame, CA).

Cells were stained for inhibin $\alpha$-subunit by treating them with an antigen-retrieval solution containing 0.1 mol sodium citrate $\mathrm{I}^{-1}, \mathrm{pH} 6$, for $5 \mathrm{~min}$ at $90^{\circ} \mathrm{C}$, and then allowing them to cool in this solution (Shi et al., 1993). The cells were washed in PBS, blocked in 10\% normal goat serum, and then incubated with $0.5 \%(\mathrm{v} / \mathrm{v})$ monoclonal anti-inhibin $\alpha$ (Cell Marque, Austin, TX) for $1 \mathrm{~h}$, followed by $1.7 \%$ (v/v) FITC anti-mouse IgG for $1 \mathrm{~h}$. Cells were mounted on to glass slides using Vectashield with 4',6-diamidino-2-phenylindole dihydrochloride (DAPI, Vector Laboratories).

\section{Flow cytometry}

Sertoli cells were analysed by flow cytometry to determine DNA content per cell as a measure of progression through the cell cycle. Cells were removed from the culture vessel

appearance of rhFSH-treated Sertoli cells 2 days after Sertoli cell aggregates were seeded on to a laminin-coated vessel (ultra-pure preparation: cells have effectively been in culture for 7 days). (c) Alkaline phosphatase histochemistry demonstrating two contaminating peritubular cells in a high-purity Sertoli cell preparation after 2 days in culture. Scale bars represent (a) $50 \mu \mathrm{m}$ and (b,c) $10 \mu \mathrm{m}$. 
by treatment with trypsin-versene solution for 5-10 min at $37^{\circ} \mathrm{C}$. Cells were pelleted by centrifugation at $300 \mathrm{~g}$ and resuspended in PBS, and then added to an equal volume of ice-cold ethanol for $10 \mathrm{~min}$. The cell suspension in ethanol was underlayered with $1 \mathrm{ml}$ PBS and centrifuged for $5 \mathrm{~min}$ at $300 \mathrm{~g}$ to rehydrate and pellet cells. The supernatant was discarded and the cells were rinsed once more in PBS before resuspension in $0.01 \%(\mathrm{w} / \mathrm{v})$ RNAse $\mathrm{A}$ (Sigma) in PBS and incubation for $10 \mathrm{~min}$ at $37^{\circ} \mathrm{C}$. An equal volume of $0.01 \%(\mathrm{w} / \mathrm{v})$ propidium iodide (PI; Sigma) was added and the cells were stored at $4^{\circ} \mathrm{C}$ for at least $1 \mathrm{~h}$ before cytometry.

Cells were measured using a MOFLO flow cytometer (Cytomation, Fort Collins, CO) with an argon ion laser operating at $488 \mathrm{~nm}$. Forward angle scatter, right angle scatter and PI fluorescence (detected through a $630 \mathrm{~nm}$ bandpass filter) were measured from at least 30000 events for each sample. Flow)o software (TreeStar software, San Carlos, CA) was used to analyse data. Events were gated by light scatter characteristics to exclude debris and cell doublets, generating sets of at least 5000 single-cell events for each sample (with the exception of samples of dying cells that had detached from the culture vessel, where gating resulted in $<500$ events). A histogram was generated for PI fluorescence (which is directly proportional to DNA content per cell) for each sample.

\section{Cell doubling time}

The Sertoli cell doubling time was approximated by loading six laminin-coated six-well culture plates with either $4 \times 10^{4}$ cells $\mathrm{cm}^{-2}$ or $2 \times 10^{5}$ cells $\mathrm{cm}^{-2}$ with ITS and with or without rhFSH. Cells were allowed to attach and start growing for 2 days before two plates were trypsinized and the cells were counted using a haemocytometer. The remaining plates were allowed to grow for a further 48 or $72 \mathrm{~h}$ before the number of cells was determined. Doubling time at each time point was determined using the equation: $t_{\text {double }}=\Delta t / \log _{2}\left(n_{2} / n_{1}\right)$, where $\Delta t$ is the time between the first and second cell counts, and $n_{1}$ and $n_{2}$ are the first and second counts, respectively.

\section{Statistical analysis}

Data are expressed as mean \pm SEM unless otherwise indicated. Comparisons between groups involved either Student's two tailed $t$ test (in the case of comparisons between two data sets), or one-way ANOVA followed by Tukey's post-hoc test (in the case of comparisons between multiple data sets). All statistical analysis was performed using Prism version 2 (Graphpad Software, San Diego, CA). Differences between groups were considered significant when $P<0.05$, and highly significant when $P<0.01$.

\section{Results}

\section{Isolation of Sertoli cells}

The inability of Sertoli cells to adhere efficiently to an uncoated polystyrene surface is the key feature of the ultra- pure method of Sertoli cell preparation. Peritubular cells bind preferentially and hence were immobilized on the uncoated polystyrene cell culture plate, whereas, when stimulated with rhFSH, most Sertoli cells formed spherical aggregates that were suspended in the medium (Fig. 1a). Sertoli cell aggregates were harvested and subcultured on to laminin-coated vessels. After $24 \mathrm{~h}$ of culture, the Sertoli cells formed a monolayer (Fig. 1b). A small number of single cells was observed to be in a plane above the Sertoli cell layer; these cells had morphological characteristics consistent with gonocytes in culture, and they disappeared from the culture over a period of 2-3 days.

The degree of peritubular cell contamination in Sertoli cell cultures was determined after 4 days by alkaline phosphatase histochemistry (Fig. 1c); high purity Sertoli cells had $1.96 \pm 1.4 \%$ ( $n=3$ separate preparations) peritubular cell contamination, whereas ultra-pure Sertoli cells (cultures that had been depleted of peritubular cells by immobilizing them on a culture plate) had $0.16 \pm 0.54 \%(n=3$ separate preparations) peritubular cell contamination (only two peritubular cells were counted in the three separate preparations). These results demonstrate that the ultra-purification method used in the present study results in an approximate tenfold decrease in peritubular cell contamination compared with published methods.

The phenotype of these cells was confirmed by immunohistochemistry for inhibin $\alpha$ (Fig. 2e,f), a marker for Sertoli cells (Schlatt et al., 1996), and $\alpha$-smooth muscle actin (Fig. 2a-d), a marker for peritubular cells (Tung and Fritz, 1990). There was no equivocacy in the characterization of cultured cells; cells that had a peritubular cell morphology (elongated and spindle shaped) always expressed alkaline phosphatase and $\alpha$-smooth muscle actin, whereas cells that had a Sertoli cell morphology (rounded with a central nucleus) always expressed inhibin $\alpha$-subunit. There was no evidence of any other type of cell after 4 days in culture.

The composition of ultra-pure cultures was assessed after 22 days in culture (that is, just before the end of the culture period) to verify that contaminating cells were not proliferating at a higher rate than Sertoli cells and overcoming the culture. In ultra-pure preparations, peritubular cell contamination had increased to $2.7 \pm 2.7 \% \quad(n=3$ preparations). Most peritubular cells were associated with each other in clonal colonies of 2-30 cells. Immunohistochemistry demonstrated that Sertoli cells remained proliferative (as assessed by BrdU incorporation; Fig. 3a,b), whereas contaminating peritubular cells were very rarely observed to incorporate BrdU. Inhibin $\alpha$ subunit expression was not as marked as it was at the start of the culture period, although it clearly labelled most of the cells in culture as Sertoli cells (Fig. 3c). No cells were observed after 22 days in culture that did not appear to be either Sertoli or peritubular cells.

Doubling time of Sertoli cells treated with rhFSH appeared to be approximately one-fifth to one-tenth of the doubling time of cells at similar density in the absence of FSH (Fig. 4c). Doubling time was strongly related to plated cell density, and the shortest doubling time observed was from 

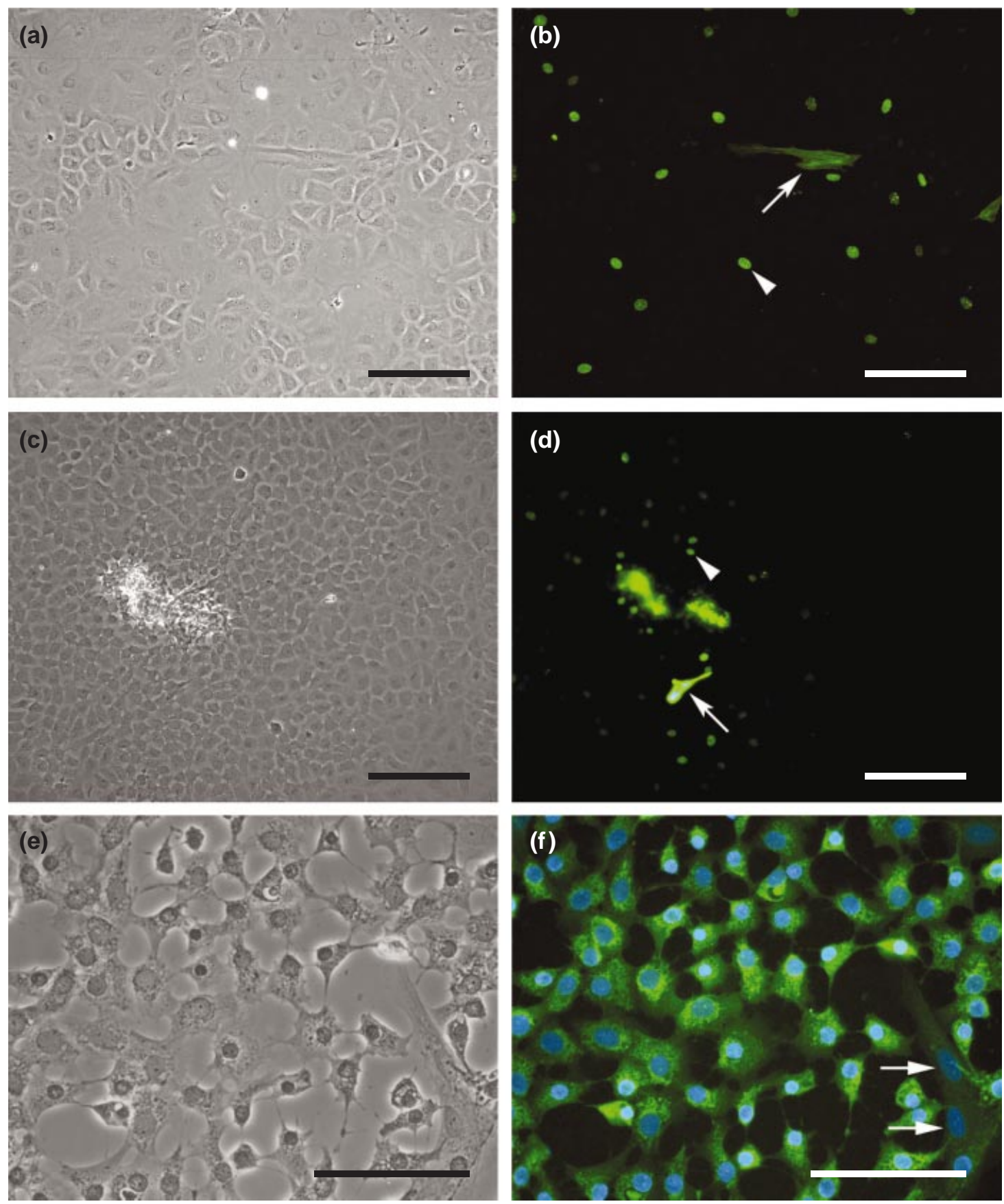

Fig. 2. Immunofluorescence of $\alpha$-smooth muscle actin, inhibin $\alpha$ subunit, and incorporated 5-bromo-2'deoxyuridine $(\mathrm{BrdU})$ in cultured high-purity preparations of rat Sertoli cells. Micrographs are typical of those observed in three separate experiments. (a) Bright-field and (b) corresponding dark-field appearance of passage 2 (day 11 after birth, corresponding to day 5 of culture) Sertoli cell preparation, demonstrating $\alpha$-smooth muscle actin (cytoplasmic fluorescence in contaminating peritubular cells; an example is indicated by the arrow), and incorporated BrdU (nuclear fluorescence indicating dividing cells, primarily Sertoli cells; an example is indicated by the arrowhead). (c) Bright-field and (d) corresponding dark-field appearance of passage 4 (day 25 after birth, corresponding to day 19 of culture) Sertoli cell preparation demonstrating $\alpha$-smooth muscle actin (cytoplasmic fluorescence in contaminating peritubular cells; an example is indicated by the arrow), and incorporated BrdU (nuclear fluorescence in dividing cells; an example is indicated by the arrowhead). (e) Bright-field and (f) corresponding dark-field appearance of passage 2 (day 10 after birth, corresponding to day 4 of culture) Sertoli cell preparation demonstrating inhibin $\alpha$. Note the punctate cytoplasmic staining and absence of staining in peritubular cells (indicated by arrows). Counterstained with 4',6-diamidino-2-phenylindole dihydrochloride (DAPI) nuclear stain. Scale bars represent $50 \mu \mathrm{m}$. 

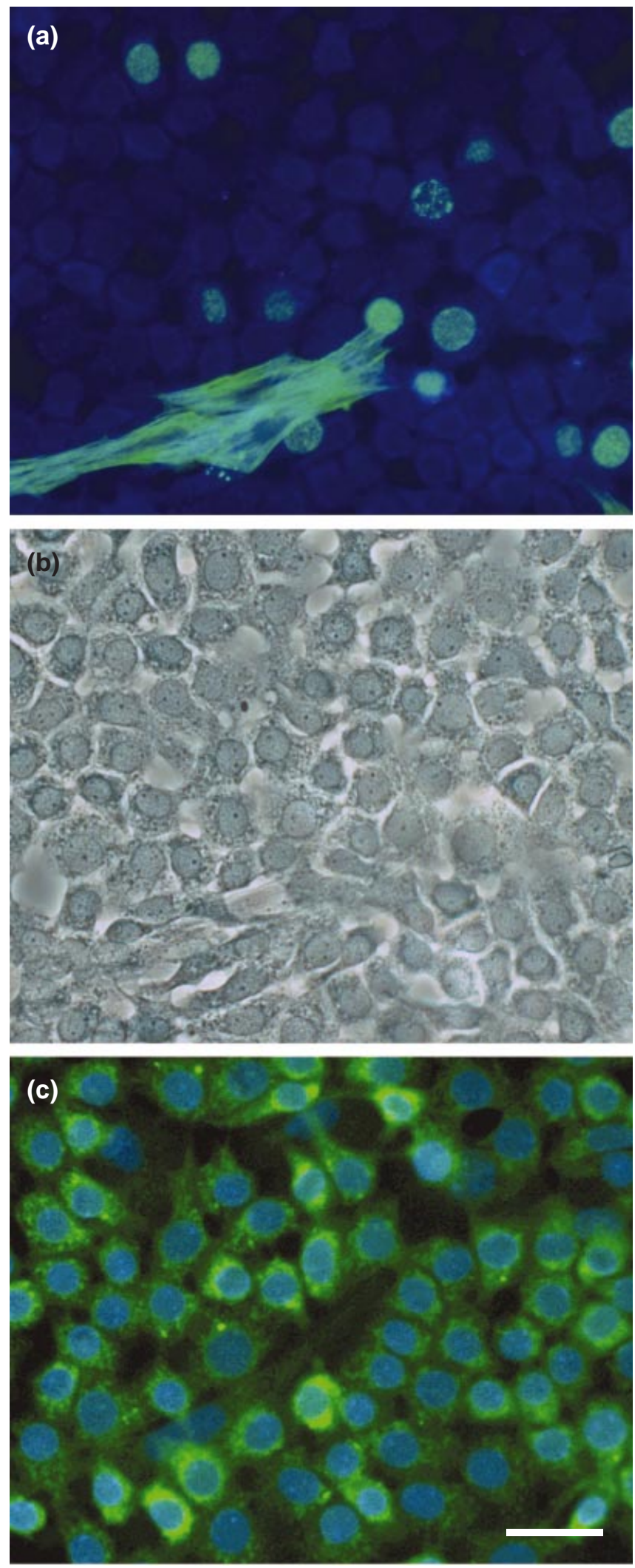

Fig. 3. Immunofluorescence of $\alpha$-smooth muscle actin, inhibin $\alpha$ subunit, and incorporated 5-bromo-2'-deoxyuridine (BrdU) in cultured ultra-pure preparations of rat Sertoli cells after 22 days
FSH-treated cells at approximately $7 \times 10^{4}$ cells $\mathrm{cm}^{-2}$, which doubled in $52 \pm 14 \mathrm{~h}$ (range 27.9-77.3). In general, rhFSHtreated cells were passaged at 1:4 (first passage after initial seeding was always 1:3, subsequent passages depended upon cell density but ranged from 1:3-1:5), and required passage approximately every 8 days. Therefore, it was concluded that these cells underwent between five and seven population doublings during their period of culture.

\section{Enhancement of proliferation}

rhFSH induced a significant increase in both the rate (an approximately 1.6-fold increase over a wide range of concentrations, Fig. 5) and duration (Fig 4b) of Sertoli cell proliferation compared with oFSH. When stimulated with oFSH, Sertoli cell proliferation had decreased to below control values after day 14 of culture (equivalent to day 20 after birth); however, stimulation with rhFSH extended the proliferative phase to beyond day 22 of culture (equivalent to day 28 after birth; Fig. 4a,b). This finding was confirmed by immunohistochemistry, which demonstrated that Sertoli cells actively incorporate BrdU, a marker for cellular proliferation, at day 5 (Fig. 2a,b) and day 19 (Fig. 2c,d) of culture, equivalent to days 11 and 25 after birth, respectively.

It was hypothesized that oFSH contains a factor that suppresses the proliferation of Sertoli cells (hence shortening the duration of the proliferative phase in vitro). This hypothesis was tested by examining the proliferation of Sertoli cells stimulated with a mixture of $\mathrm{OFSH}$ and rhFSH. The presence of oFSH in rhFSH media resulted in a proliferative phase similar in duration to that observed with oFSH media alone (Fig. 4b), strongly indicating the presence of an anti-proliferative or pro-differentiation factor in the oFSH preparation.

Cells were analysed by flow cytometry at day 3 , day 12 and day 22 of culture (equivalent to day 9, day 18 and day 28 after birth, respectively; Fig. 6a-c) to determine DNA content per cell. A computational algorithm (Watson et al., 1987) was used to analyse G0-G1-, and G2-M-phase peaks and S-phase populations. This algorithm was constrained such that the G2-M-phase peak was at a position of twice

in culture. (a) Immunofluorescence of $\alpha$-smooth muscle actin and incorporated $\mathrm{BrdU}$ in an ultra-pure preparation of Sertoli cells. Green nuclear fluorescence is incorporated BrdU, which labels S-phase cells. Green cytoplasmic fluorescence is $\alpha$-smooth muscle actin, which labels contaminating peritubular cells (three are shown in this field). Blue staining is 4',6-diamidino-2phenylindole dihydrochloride (DAPI) counterstain, which becomes less effective (and hence perinuclear, rather than nuclear) as a result of the preparation methods required for BrdU immunofluorescence. (b) Bright-field photomicrograph corresponding to the immunofluorescent photomicrograph shown in (a). (c) Immunofluorescent demonstration of inhibin $\alpha$-subunit in an ultrapure preparation of Sertoli cells. Green, punctate cytoplasmic stain is inhibin $\alpha$-subunit in Sertoli cells. Blue staining is DAPI nuclear counterstain. Scale bar represents $20 \mu \mathrm{m}$. 

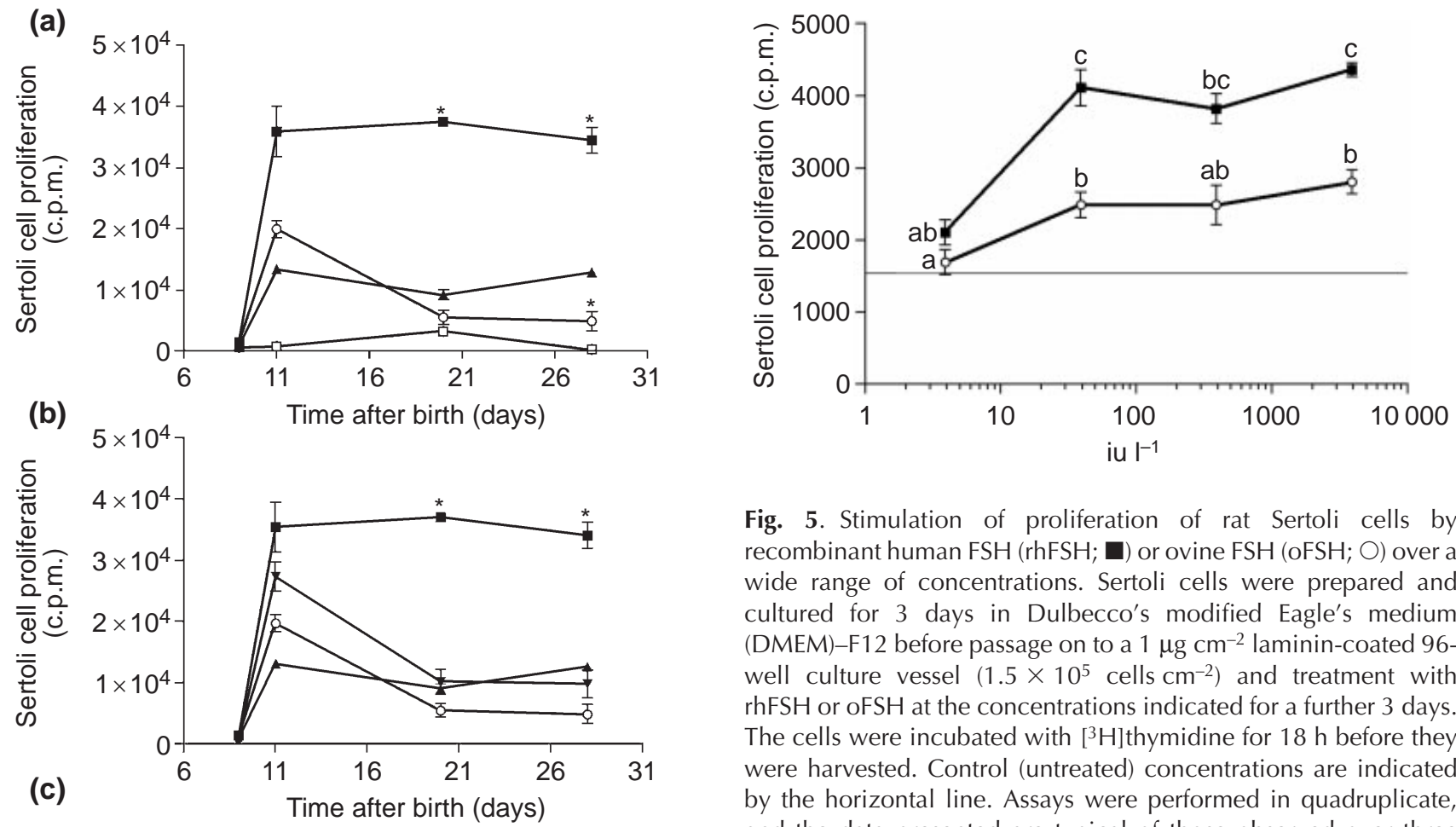

Fig. 5. Stimulation of proliferation of rat Sertoli cells by recombinant human $\mathrm{FSH}(\mathrm{rhFSH} ; \mathbf{\square})$ or ovine $\mathrm{FSH}(\mathrm{oFSH} ; \mathrm{O})$ over a wide range of concentrations. Sertoli cells were prepared and cultured for 3 days in Dulbecco's modified Eagle's medium (DMEM)-F12 before passage on to a $1 \mu \mathrm{g} \mathrm{cm}^{-2}$ laminin-coated 96well culture vessel $\left(1.5 \times 10^{5}\right.$ cells $\left.\mathrm{cm}^{-2}\right)$ and treatment with rhFSH or oFSH at the concentrations indicated for a further 3 days. The cells were incubated with $\left[{ }^{3} \mathrm{H}\right]$ thymidine for $18 \mathrm{~h}$ before they were harvested. Control (untreated) concentrations are indicated by the horizontal line. Assays were performed in quadruplicate, and the data presented are typical of those observed over three separate experiments. ${ }^{\mathrm{a}-\mathrm{c} S i g n i f i c a n t}$ differences are indicated by different superscripts $(P<0.05)$.

characteristics of this model is shown (Fig. 6f). The proportion of cells calculated to be in each phase of the cell cycle was similar throughout the culture period, demonstrating that Sertoli cell proliferation was similar throughout the culture period.

After days 26-32 of culture (equivalent to days 32-38 after birth), Sertoli cells had assumed an abnormal morphology that included a large number of cytoplasmic vacuoles close to the nucleus, a very large cytoplasmic area, and often the appearance of more than one nucleus. Flow cytometry indicated an accumulation of cells in G0-G1 phase (Fig. 6d). These observations are consistent with the cells having undergone classical senescence (or 'culture shock'; Sherr and DePinho, 2000). After 1-2 days, the cells detached from the culture vessel and flow cytometry indicated a variable DNA content per cell (Fig. 6e) consistent with cells undergoing apoptosis.

\section{Discussion}

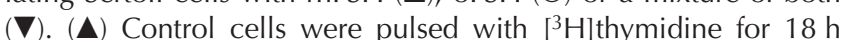
before they were harvested at the time points indicated. (c) Sertoli cell doubling time was determined from cells at various densities and treated with $(\mathbf{\square})$ or without $(\mathbf{\Lambda})$ rhFSH. ${ }^{\text {a-c Significant differences }}$ $(P<0.05)$ are indicated by superscripts that differ by a single letter. Asterisks indicate significant differences from the control $(\mathbf{\Lambda})(P<0.05)$.

the G0-G1-phase peak (that is, G2-M-phase cells contained twice as much DNA as G0-G1-phase cells) to ensure a good fit. A representation of the curve-fitting

The results of the present study demonstrate that, given an appropriate culture environment, Sertoli cells may proliferate in vitro well beyond their normal proliferative phase in vivo. This extension of the proliferative phase is achieved by minimizing the number of contaminating peritubular cells in the preparation, removing inhibitors of Sertoli cell proliferation, and culturing in the absence of factors, such as thyroid hormone, that are known to induce Sertoli cell differentiation. The doubling time of FSH-treated 

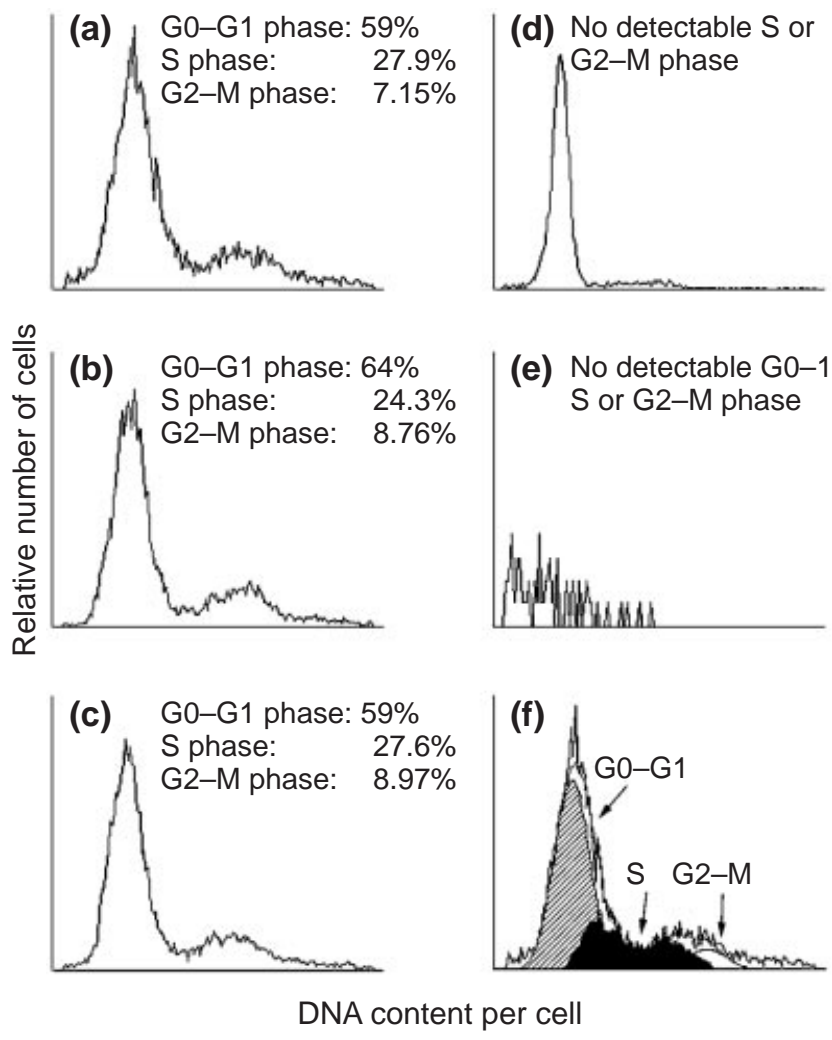

Fig. 6. Cell cycle profiles of cultured rat Sertoli cells after analysis using flow cytometry. DNA content per cell was measured in highpurity Sertoli cells stimulated with recombinant human FSH (rhFSH). Cells were plated at $3 \times 10^{4}$ cells $\mathrm{cm}^{-2}$ on laminin-coated culture vessels and grown for 5 days before passage. This procedure was repeated until the cells ceased proliferation. Cells were analysed at days (a) 3, (b) 12, (c) 22 and (d) 32 of culture (equivalent to days 9, 18, 28 and 38 after birth, respectively). (e) At day 35 of culture (equivalent to day 41 after birth), most cells had detached from the culture vessel and these detached cells were also analysed. (f) Computational model (Watson pragmatic model) used to find G0-G1-, S- and G2-M-phase populations. The calculated populations are superimposed on the input histogram of cells analysed at day 3 of culture. 娄: G0-G1-phase distribution;

口: S-phase population; $\square$ : G2-M-phase population.

Sertoli cells in this culture system at relatively low density is $52 \pm 14 \mathrm{~h}$, which is comparable to the average doubling time of $44 \mathrm{~h}$ between day 5 and day 10 after birth in vivo (Wang et al., 1989). Rat Sertoli cells undergo only 2.5 population doublings after day 5 after birth in vivo (Wang et al., 1989); however, in the culture system used in the present study, calculations indicate that Sertoli cells can undergo between five and seven population doublings. Cell doubling time was found to be highly dependent on cell density, consistent with findings by Schlatt et al. (1996) indicating contact inhibition of proliferation.

Investigators in this field of study have often referred to the similarities between the duration of proliferation of Sertoli cells in vitro and in vivo. These similarities appear now to be artefactual. The propagation of Sertoli cells in culture represents a system that is far from physiological and that is deficient in several important endocrine factors. The most obvious of these factors is thyroid hormone, which regulates the timing of Sertoli cell differentiation in vivo. When hypothyroidism is induced in neonatal rats, Sertoli cell proliferation is extended until about day 30 after birth (Cooke and Meisami, 1991; Van Haaster et al., 1992). Given that, in general, cultured Sertoli cells are maintained in the complete absence of thyroid hormone, these cells would be expected to proliferate for considerably longer than the 10-12 days after birth that has been accepted in the literature. The present study demonstrates that Sertoli cells in vitro are, in fact, capable of proliferating for a period comparable to that of hypothyroid Sertoli cells in vivo.

The oFSH preparation contains a factor or factors that negatively regulate Sertoli cell proliferation, as addition of oFSH to rhFSH media suppressed the proliferative phase of Sertoli cells to a duration similar to that observed in Sertoli cells stimulated with oFSH alone. In addition to the increased duration of proliferation demonstrated using $\mathrm{rhFSH}$, the apparent rate of Sertoli cell proliferation was also increased. Factors present in the oFSH preparation may progressively initiate differentiation of Sertoli cells, in effect reducing the total pool of dividing cells and resulting in a decrease in the rate of thymidine incorporation. The factor or factors in oFSH that produce this effect may be copurified hormones, proteases, chemical byproducts from the purification process, or different isoform compositions between oFSH and rhFSH.

This paper reports a number of modifications to the purification and culture of dividing Sertoli cells that result in a substantial increase in the duration of the proliferative phase, providing a valuable experimental model for investigators studying the control of Sertoli cell division. A major implication of these studies is the finding that the timing of Sertoli cell differentiation is not totally dictated by an intrinsic timing mechanism. Instead, external signals must act on these cells to initiate timely differentiation. In the absence of these external signals, the cell continues to divide well beyond the normal in vivo proliferative phase, until ultimately it is restricted by an internal mechanism.

The authors gratefully acknowledge the technical assistance of P. Hutchinson. The authors would also like to thank A. Parlow of the NIDDK at NIH for providing the ovine FSH, and M. O'Bryan, M. Hedger, G. Risbridger, A. O'Connor and D. de Kretser for helpful discussions. These studies were supported by the Australian Research Council (ARC grant number A09927208).

\section{References}

Boitani C, Stefanini M, Fragale A and Morena AR (1995) Activin stimulates Sertoli cell proliferation in a defined period of rat testis development Endocrinology 136 5438-5444

Chapin RE, Phelps JL, Miller BE and Gray TJ (1987) Alkaline phosphatase histochemistry discriminates peritubular cells in primary rat testicular cell culture Journal of Andrology 8 155-161 
Cooke PS and Meisami E (1991) Early hypothyroidism in rats causes increased adult testis and reproductive organ size but does not change testosterone levels Endocrinology 129 237-243

Meachem SJ, McLachlan RI, de Kretser DM, Robertson DM and Wreford NG (1996) Neonatal exposure of rats to recombinant follicle stimulating hormone increases adult Sertoli and spermatogenic cell numbers Biology of Reproduction 54 36-44

Orth JM (1982) Proliferation of Sertoli cells in fetal and postnatal rats: a quantitative autoradiographic study Anatomical Record 203 485-492

Orth JM (1984) The role of follicle-stimulating hormone in controlling Sertoli cell proliferation in testes of fetal rats Endocrinology 115 1248-1255

Orth JM, Gunsalus GL and Lamperti AA (1988) Evidence from Sertoli celldepleted rats indicates that spermatid number in adults depends on numbers of Sertoli cells produced during perinatal development Endocrinology 122 787-794

Risbridger GP, Hancock A, Robertson DM, Hodgson Y and de Kretser DM (1989) Follitropin (FSH) stimulation of inhibin biological and immunological activities by seminiferous tubules and Sertoli cell cultures from immature rats Molecular and Cellular Endocrinology 67 1-9

Schlatt S, de Kretser DM and Loveland KL (1996) Discriminative analysis of rat Sertoli and peritubular cells and their proliferation in vitro: evidence for follicle-stimulating hormone-mediated contact inhibition of Sertoli cell mitosis Biology of Reproduction 55 227-235

Sherr CJ and DePinho RA (2000) Cellular senescence: mitotic clock or culture shock? Cell 102 407-410

Shi S, Chaiwun B, Young L, Cote R and Taylor C (1993) Antigen retrieval technique utilizing citrate buffer or urea solution for immunohistochemical demonstration of androgen receptor in formalin-fixed paraffin sections Journal of Histochemistry and Cytochemistry 41 1599-1604
Steinberger A and Steinberger E (1971) Replication pattern of Sertoli cells in maturing rat testis in vivo and in organ culture Biology of Reproduction 4 84-87

Steinberger A, Heindel JJ, Lindsey JN, Elkington JS, Sanborn BM and Steinberger E (1975) Isolation and culture of FSH responsive Sertoli cells Endocrine Research Communications 2 261-272

Tung PS and Fritz IB (1990) Characterization of rat testicular peritubular myoid cells in culture: alpha-smooth muscle isoactin is a specific differentiation marker Biology of Reproduction 42 351-365

Van Haaster LH, De Jong FH, Docter R and De Rooij DG (1992) The effect of hypothyroidism on Sertoli cell proliferation and differentiation and hormone levels during testicular development in the rat Endocrinology 131 1574-1576

van Haaster LH, de Jong FH, Docter R and de Rooij DG (1993) High neonatal triiodothyronine levels reduce the period of Sertoli cell proliferation and accelerate tubular lumen formation in the rat testis, and increase serum inhibin levels Endocrinology 133 755-760

Wang ZX, Wreford NG and De Kretser DM (1989) Determination of Sertoli cell numbers in the developing rat testis by stereological methods International Journal of Andrology 12 58-64

Watson JV, Chambers SH and Smith PJ (1987) A pragmatic approach to the analysis of DNA histograms with a definable G1 peak Cytometry 8 1-8

Received 19 April 2002.

First decision 3 July 2002.

Revised manuscript received 8 July 2002.

Accepted 1 August 2002. 